Bull. Austral. Math. Soc.

20D25, 20D15

VOL. 42 (1990) [499-510]

\title{
THE WIELANDT SUBGROUP OF METACYCLIC P-GROUPS
}

\author{
Elizabeth A. Ormerod
}

The Wielandt subgroup is the intersection of the normalisers of all the subnormal subgroups of a group. For a finite group it is a non-trivial characteristic subgroup, and this makes it possible to define an ascending normal series terminating at the group. This series is called the Wielandt series and its length determines the Wielandt length of the group. In this paper the Wielandt subgroup of a metacyclic $p$-group is identified, and using this information it is shown that if a metacyclic p-group has Wielandt length $n$, its nilpotency class is $n$ or $n+1$.

\section{INTRODUCTION}

The subgroup of a group that has become known as the Wielandt subgroup was originally defined by Helmut Wielandt in a paper published in 1958 [5]. As he showed, the Wielandt subgroup $\omega(G)$ of a group $G$, defined by

$$
\omega(G)=\bigcap_{H \triangleleft \triangleleft G} N_{G}(H)
$$

is a characteristic, non-trivial subgroup of $G$ for any finite group $G$. It is possible to define a normal series, the Wielandt series, for a group $G$, in a similar way to the upper central series. We put

$$
\omega_{0}(G)=1, \omega_{i}(G) \triangleleft G \text { such that } \omega_{i}(G) / \omega_{i-1}(G)=\omega\left(G / \omega_{i-1}(G)\right)
$$

for $i \geqslant 1$. Since $\omega(G)$ is non-trivial, for a finite group $G$ there is an integer $n$ such that $\omega_{n}(G)=G$. We call the smallest such $n$ the Wielandt length of $G$.

When $G$ is nilpotent, $\omega(G)$ is the intersection of the normalisers of all subgroups and coincides with the norm of a group (see [3]). The Wielandt subgroup will always contain the centre of a group, and Schenkman [3] has shown that the norm is in the second centre of a group. For a nilpotent group $G$, we have

$$
\zeta_{1}(G) \leqslant \omega(G) \leqslant \zeta_{2}(G)
$$

Received 9 January 1990

This research was supported by a grant from the Australian Research Council.

Copyright Clearance Centre, Inc. Serial-fee code: 0004-9729/90 \$A2.00+0.00. 
This implies that if a nilpotent group has Wielandt length $n$, then its class is between $n$ and $2 n$. Beyond this little is known about the relationship between the Wielandt length of a nilpotent group and its class, although Camina [1] has looked briefly at the influence of the Wielandt length on the structure of a p-group.

The aim of this paper is to identify the Wielandt subgroup of a metacyclic $p$ group, and using this to establish a relationship between the Wielandt length and the class. The identification of the Wielandt subgroup in this case relies fairly heavily on the presentation of the metacyclic $p$-group. Such presentations have been classified by King in 1973 [2]. More recently Newman and Xu have produced presentations which have been helpful in this work. Their results for $p$ odd are announced in [4], while further details, including the case for $p=2$, will appear later. Once the Wielandt subgroup is identified it is possible to show that for a metacyclic p-group with Wielandt length $n$, the class is either $n$ or $n+1$. Since there is evidence that this is true for other small groups, it is tempting to suggest that this might be true for all nilpotent groups. However, it is not the case as a group has been found that violates this relationship.

\section{NOTATION AND PRELIMINARY RESULTS}

In general, notation is standard. The prime under consideration will be denoted by $p$. In particular,

$G^{\prime}$ is the derived group of a group $G$;

$1, \zeta_{1}(G), \ldots, \zeta_{i}(G), \ldots$ is the upper central series of a group $G$

$p^{k} \| w$ means $p^{k} \mid w$ and $p^{k+1} \nmid w$.

The following commutator identities are standard and will be used without explicit reference:

$$
[x y, z t]=[x, t]^{y}[y, t][x, z]^{y t}[y, z]^{t}
$$

and hence

$$
\left[x^{-1}, z\right]=[z, x]^{x^{-1}}, \quad\left[x, y^{-1}\right]=[y, x]^{y^{-1}} .
$$

The following identities hold in any metabelian group and will also be used extensively. For any positive integer $k$,

$$
\left[v, w^{k}\right]=\prod_{1 \leqslant i \leqslant k}[v, i w]^{\left(\begin{array}{l}
k \\
i
\end{array}\right)}
$$

and

$$
\left.\left(v w^{-1}\right)^{k}=v^{k}\left(\prod_{0<i+j<k}[v, i w, j v]^{(i+j+1}{ }^{k}\right)\right) w^{-k}
$$


The presentations of metacyclic p-groups have been classified by King [3]. We follow this classification as it has been further developed by Newman and Xu [4]. For $p \neq 2$ any metacyclic $p$-group has a unique presentation of the form

$$
G=\left\langle a, b: a^{p^{r+a+u}}=1, b^{p^{r+a+t}}=a^{p^{r+q}}, a^{b}=a^{1+p^{r}}\right\rangle
$$

where $r \geqslant 1, u, s, t \geqslant 0$ and $u \leqslant r . G$ is a split extension if and only if $u=0$ or $s=0$ or $t=0$. For $p=2$ the situation is more complex and we treat this later.

As a consequence of the relations of the group $G$, and the identity 2.1 , the following identities hold in $G$. From here, until otherwise stated, it will be assumed that $p$ is an odd prime.

(2.4) For any positive integer $i$,

(i) $[a, i b]=a^{p^{i r}}$;

(ii) $\left[a^{i}, b\right]=[a, b]^{i}$;

(iii) $\left[a, b^{i}\right]^{p^{\mathbf{a}}}=[a, b]^{i p^{\boldsymbol{*}}}$.

From King we state the following results:

(2.5) $[2,2.1 \mathrm{a}]$. Let $k$ be an integer, $k \geqslant 0$. Then $p^{k} \| w$ implies that $p^{k+r} \|$ $\left(1+p^{r}\right)^{w}-1$.

(2.6) $[2,4.1]$. For integers $\alpha, \beta, \lambda, k$, with $k \geqslant 1$,

$$
\left(a^{\alpha} b^{\beta}\right)^{p^{k}}=a^{\alpha p^{k}} b^{\beta p^{k}}\left[a^{\alpha}, b^{\beta}\right]^{\lambda p^{k-1}}
$$

where $p \mid \lambda$ if and only if $p \geqslant 3$.

(2.7) $[2,4.10(\mathrm{ii})]$. The centre of $G$ is given by

$$
\zeta_{1}(G)=\left\langle a^{p^{p+u}}, b^{p^{p+u}}\right\rangle .
$$

From these results we derive several other identities which are quite crucial.

(i) $\left[a, b^{p+t}\right]=[a, b]^{p+t}=a^{p^{r+o+t}}$

(ii) $(b a)^{p^{r+c+t}}=b^{p^{r+c+t}} a^{p^{r+s+c}}$

(iii) $(b a)^{p+c+u}=b^{p+c+u} a^{p+c+u}=b^{p+c+u}$.

\section{IDENTIFICATION OF THE WIELANDT SUBgroup}

We have seen that for a nilpotent group the Wielandt subgroup can be described as the intersection of the normalisers of all subgroups. To make computation easier 
we aim to reduce the number of intersections that need to be found. We get a small reduction by noting that it is sufficient to find the intersection of the normalisers of all cyclic groups. However, this is still unwieldy, and we are able to make the following reduction.

TheOREM 3.1. Let $G$ be a metacyclic p-group, with presentation given in (2.3). Then

$$
\omega(G)=N_{G}(\langle b\rangle) \cap N_{G}\left(\left\langle b^{p^{t}} a^{-1}\right\rangle\right) .
$$

Proof: The proof in one direction is trivial. For the non-trivial direction we assume that $w$ is a fixed element of $G, w \in N_{G}(\langle b\rangle) \cap N_{G}\left(\left\langle b^{p} a^{-1}\right\rangle\right)$. Some direct consequences are:

(1) Since $w$ normalises $\langle b\rangle$, then $[w, b] \in\langle b\rangle \cap G^{\prime}=\left\langle a^{p^{r+a}}\right\rangle$. Hence $b^{w}=$ $b a^{m p^{r+s}}=b^{1+m p^{r+0+t}}$ for some integer $m$.

(2) We note that using (2.6) the element $x=b^{p^{t}} a^{-1}$ has order $p^{r+s}$ and any element of $\langle x\rangle$ can be written $b^{k p^{i}} a^{i}$ where $1 \leqslant k<p^{r+\boldsymbol{t}}$. The value of $i$ is not unrestricted, but it is not important here. In particular, $\langle x\rangle \cap\langle a\rangle=1$, and hence $\langle x\rangle \cap G^{\prime}=1$. Thus, since $w$ normalises $\langle x\rangle$, we have $[w, x] \in(x) \cap G^{\prime}=1$, and so $\left(b^{p^{t} a^{-1}}\right)^{w}=b^{p^{t}} a^{-1}$.

(3) The group $\langle a\rangle$ is normal in $G$, but we can now be quite specific about the action of $w$ on $a$. Since $a=\left(b^{p^{t}} a^{-1}\right)^{-1} b^{p^{t}}$, we have $a^{w}=a^{1+m p^{r+c+c}}$.

We now show that $w \in N_{G}(\langle g\rangle)$ for any $g \in G$. This is a sufficient condition for $w$ to belong to $\omega(G)$. For any $g \in G, g$ can be written $g=b^{j} a^{i}$ where $i$ and $j$ are non-negative integers. Then

$$
\begin{aligned}
& g^{w}=\left(b^{j} a^{i}\right)^{w} \\
& =\left(b^{w}\right)^{j}\left(a^{w}\right)^{i} \\
& =\left(b^{1+m p^{r+a+t}}\right)^{j}\left(a^{1+m p^{r+a+t}}\right)^{i} \\
& =b^{j} a^{i} b^{j m p^{r+s+t}} a^{i m p^{r+c+t}} \quad \text { since } b^{m p^{r+\imath+t}}, a^{p^{r+s+t}} \in \zeta(G) \\
& =b^{j} a^{i}\left(b^{j} a^{i}\right)^{m p^{r+o+t}} \quad \text { by (2.8) (ii) } \\
& =\left(b^{j} a^{i}\right)^{1+m p^{r+o+t}} \text {. }
\end{aligned}
$$

This gives the desired result.

We are now able to prove the main theorem of this section.

ThEOREM 3.2. Let $G$ be a metacyclic p-group, with presentation given in (2.3). Then

(i) for $0 \leqslant u \leqslant t$,

$$
\omega(G)=\left\langle a^{p^{*}}, b^{p^{s+u}}\right\rangle
$$


(ii) for $u>t \geqslant 0$,

$$
\omega(G)=\left\langle a^{p^{u-t+\varepsilon}}, b^{p^{t+\varepsilon} a^{-p^{t}}}\right\rangle .
$$

Proof: We let $w=b^{j} a^{i}$ be a member of $G$ and, using 3.1, determine precise conditions on $i$ and $j$ for $w$ to belong to $\omega(G)$.

For $w$ to normalise $\langle b\rangle$ we must have $[w, b] \in\left\langle a^{p^{r+a}}\right\rangle$. Using the usual commutator identities we have

$$
[w, b]=\left[b^{j} a^{i}, b\right]=\left[a^{i}, b\right]=a^{i p^{r}}
$$

and $a^{i p^{r}} \in\left\langle a^{p^{r+d}}\right\rangle$ if and only if $i=e p^{*}$ for some integer $e$.

Then $w$ must centralise $b^{p^{t}} a^{-1}$, and assuming $w$ normalises $\langle b\rangle$ and using standard commutator identities we have

$$
\begin{aligned}
{\left[w, b^{p^{t}} a^{-1}\right] } & =\left[b^{j} a^{e p^{p}}, b^{p^{t}} a^{-1}\right] \\
& =\left[b^{j}, a^{-1}\right]\left[a^{e p^{p}}, b^{p^{t}}\right] \\
& =\left[a, b^{j}\right][a, b]^{e p^{s+t}} \quad \text { using (2.4) (ii) and (iii) } \\
& =a^{-1} a^{\left(1+p^{r}\right)^{j}} a^{e p^{r+o+t}} .
\end{aligned}
$$

We consider two cases.

(i) $0 \leqslant u \leqslant t$.

In this case $a^{e p^{r+s+t}}=1$, and $\left[w, b^{p^{t}} a^{-1}\right]=1$ if and only if $a^{-1+\left(1+p^{r}\right)^{j}}=1$. By (2.5) this is true if and only if $p^{s+u} \mid j$. So we must have $w=b^{d p^{a+u}} a^{e p^{*}}$ where $d$ and $e$ are integers. Since $b^{p^{++u}} \in \zeta_{1}(G), w \in \omega(G)$ if and only if

$$
w \in\left(a^{p^{p}}, b^{p^{p+u}}\right)
$$

which is the required result.

(ii) $u>t \geqslant 0$.

In this case $\left[w, b^{p^{t}} a^{-1}\right]=1$ if and only if $a^{-1+\left(1+p^{r}\right)^{j}}=a^{e p^{r+t+t}}=1$, or $1 \neq$ $a^{-1+\left(1+p^{r}\right)^{j}}=a^{-e p^{r+c+t}}$. In the former case, $a^{-1+\left(1+p^{r}\right)^{j}}=1$ if and only if $j=d p^{s+u}$ for some integer $j$, and $a^{e p+t+t}=1$ if and only if $e=f p^{u-t}$ for some integer $e$. So we must have $w=b^{d p^{p+u}} a^{f p^{u-t+\bullet}}$ for integers $d$ and $f$. Hence, since $b^{p^{p+u}} \in \zeta_{1}(G)$, $w$ satisfies this condition if and only if

$$
\omega \in\left\langle a^{p^{u-t+s}}, b^{p^{p+u}}\right\rangle \text {. }
$$

The alternative condition is that $a^{-1+\left(1+p^{r}\right)^{j}}=a^{-e p^{r+c+t}}$. Using (2.5) again, we see that $a^{-1+\left(1+p^{r}\right)^{j}} \in\left\langle a^{p^{r+s+t}}\right\rangle$ if and only if $p^{s+t} \mid j$. So we assume $j=k p^{s+t}$ for some integer $k$. But then $a^{-1+\left(1+p^{r}\right)^{j}}=a^{k p^{r+s+t}}$ and

$$
\left[w, b^{p^{t}} a^{-1}\right]=a^{k p^{r+s+t}} a^{e p^{r+s+t}} .
$$


Hence $\left[w, b^{p^{t}} a^{-1}\right]$ is trivial if and only if $k=-e \bmod p^{u-t}$. So we must have $k=$ $-e+n p^{u-t}$ for some integer $n$, which gives $w=b^{-e p^{n+t}} a^{e p^{d}} b^{n p^{p+u}}$. We note that $b^{e p^{s+z}} a^{-e p^{q}}=\left(b^{p^{t+s}} a^{-p^{z}}\right)^{e} \bmod \left\langle a^{p^{r+a+z}}\right\rangle$.

Combining this with the previous condition, we conclude that for $u>t \geqslant 0$, $w \in \omega(G)$ if and only if

$$
w \in\left\langle a^{p^{u-t+\varepsilon}}, b^{p^{u+\varepsilon}}, b^{p^{t+d}} a^{-p^{q}}\right\rangle=\left\langle a^{p^{u-t+d}}, b^{p^{t+\varepsilon}} a^{-p^{q}}\right\rangle .
$$

This completes the proof of the theorem.

We conclude this section by pointing out that $\omega(G)=\zeta_{1}(G)$ only for the case $u=0$. For none of these groups is it the case that $\omega(G)=\zeta_{2}(G)$.

\section{The Wielandt Length of a Metacyclic $p$-Group}

Having identified that Wielandt subgroup of a metacyclic p-group, we turn our attention to the question of the relationship between the nilpotency class of the netacyclic group $G$ and the Wielandt length of $G$.

We begin by quoting a result from King.

Lemma 4.1. [2, 4.10(i)] $A$ metacyclic group $G_{1}$ with presentation given in 2.3 has nilpotency class $k$ if and only if

$$
k r \geqslant r+s+u>(k-1) r .
$$

We also note, that given a metacyclic group

$$
H=\left\langle a, b: a^{p^{\alpha}}=1, b^{p^{p}}=a^{p^{\gamma}}, a^{b}=a^{1+p^{c}}\right\rangle
$$

with $\gamma \leqslant \varepsilon, \gamma \leqslant \beta, \varepsilon+\gamma \geqslant \alpha, \varepsilon+\beta \geqslant \alpha$ then $(b)$ is normal in $H$, and we can write $H$ as a splitting extension with generators $b$ and $a_{1}=b^{\beta-\gamma} a^{-1}$ (see $[2,4.3]$ ).

We can now derive our first result.

Lемма 4.2. If the metacyclic group $G$, with presentation given in (2.3), is a split extension, of nilpotency class $k+1$, then $G / \omega(G)$ has class $k$ and is also a split extension.

Proof: As noted earlier, $G$ splits if and only if $u=0$ or $s=0$ or $t=0$. We consider these cases separately.

(i) $u=0$.

In this case $\omega(G)=\left\langle a^{p^{p}}, b^{p^{*}}\right\rangle$ and hence

$$
G / \omega(G) \cong\left\langle a, b: a^{p^{a}}=1, b^{p^{a}}=1, a^{b}=a^{1+p^{r}}\right\rangle .
$$


$G$ has class $k+1$ implies that

$$
(k+1) r \geqslant r+s>k r
$$

which is equivalent to

$$
k r \geqslant s>(k-1) r
$$

and this implies that $G / \omega(G)$ has class $k$.

(ii) $\boldsymbol{t}=\mathbf{0}$.

Here $\omega(G)=\left\langle a^{p^{u+s},}, b^{p^{s}} a^{-p^{p}}\right\rangle$ and hence

$$
G / \omega(G) \cong\left\langle a, b: a^{p^{u+s}}=1, b^{p^{b}}=a^{-p^{b}}, a^{b}=a^{1+p^{r}}\right\rangle,
$$

and again since $G$ has class $k+1, G / \omega(G)$ has class $k$. It is easily seen that $G / \omega(G)$ splits if written with generators $a$ and $b a^{-1}$.

(iii) $s=0$.

In this case, for $u>0, G$ has class $2, G \neq \omega(G)$. Hence $G / \omega(G)$ is abelian and is also a split extension.

The following corollary is immediate.

Corollary 4.3. If the metacyclic p-group $G$ is a split extension, then the Wielandt length of $G$ is the same as its nilpotency class.

The next result is a little more complex.

Lemma 4.4. Let $G$ be a metacyclic p-group with presentation as given in 2.3 and $0<u \leqslant t$. If $G$ has class $k+1$, then

(i) $G / \omega(G)$ has class $k$ if $s>(k-1) r$;

(ii) $G / \omega(G)$ has class $k-1$ if $s \leqslant(k-1) r$.

In either case $G / \omega(G)$ is a splitting extension.

Proof: From (3.2)(i), $\omega(G)=\left\langle a^{p^{p}}, b^{p^{p+u}}\right\rangle$ and hence

$$
G / \omega(G) \cong\left\langle a, b: a^{p^{b}}=1, b^{p^{+u}}, a^{b}=a^{1+p^{r}}\right\rangle .
$$

If $G$ has class $k+1$, then $(k+1) r \geqslant r+s+u>k r$. Hence $k r \geqslant s+u>(k-1) r$, and since $u \leqslant r, k r>s>(k-2) r . G / \omega(G)$ has class $k$ if $s>(k-1) r$ and class $(k-1)$ if $s \leqslant(k-1) r$. In either case, it is clear that $G / \omega(G)$ splits.

We combine this result with Corollary 4.3 to get the next corollary.

Corollary 4.5. Let $G$ be as given in Lemma 4.4, with class $k+1$. Then $G$ has Wielandt length $k+1$ if $s>(k-1) r$, and Wielandt length $k$ if $s \leqslant(k-1) r$.

The final case we need to consider is even more complex. We have

$$
G=\left\langle a, b: a^{p^{r+c t u}}=1, b^{p^{r+a+t}}=a^{p^{r+d}}, a^{b}=a^{1+p^{r}}\right\rangle
$$


where $r, s, u, t>0, u \leqslant r$ with the added condition $u>t>0$.

Let $i_{1}$ be the smallest integer such that $u-i_{1} t \leqslant t$; let $i_{2}$ be the smallest integer such that $s \leqslant i_{2} r$. Put $I=\min \left\{i_{1}, i_{2}\right\}$.

(4.6) For $0 \leqslant j \leqslant I$ define

$$
G_{j}=\left\langle a, b: a^{p^{r+a+u-j t-j r}}=1, b^{p^{r+a+t-j r}}=a^{p^{r+c-j r}}, a^{b}=a^{1+p^{r}}\right\rangle .
$$

The conditions on $I$ ensure that for $0 \leqslant j<I$,

$$
r+s+u-j t-j r>r+s+t-j r>r+s-j r>r
$$

and hence $\omega\left(G_{j}\right)$ can be found by applying Theorem 3.2 (ii). Note that $G_{0}=G$, $G_{j} \cong G_{j-1} / \omega\left(G_{j-1}\right) \cong G / \omega_{j}(G)$ for $0<j \leqslant I$.

We can now state our final result.

Lemma 4.7. With $G$ described as above, if $G$ has nilpotency class $k+1$, then the Wielandt length of $G$ is $k$ or $k+1$.

Proof: Since $G$ has class $k+1$, we have $(k+1) r \geqslant r+s+u>k r$. We consider first the case $r+s>k r$. Then, using the above notation, for $0 \leqslant j<i_{1}$ we have $u-j t>t$ and so

$$
(k+1) r \geqslant r+s+u-j t>r+s+t>r+s>k r .
$$

This implies

$$
(k-j+1) r \geqslant r+s+u-j(t+r)>(k-j) r
$$

which in turn implies that $G_{j}$ has class $k-j+1$ for $0 \leqslant j<I$. Further, if $I=i_{2}<i_{1}$, then (4.8) also implies that $G_{I}$ has class $k-I+1$. Also, since $r+s-i_{2} r \leqslant r, G_{I}$ is a split extension. Therefore, by Lemma 4.2, the Wielandt length of $G_{I}$ is $k-I+1$, and hence the Wielandt length of $G$ is $k+1$.

If $I=i_{1}$, then sitice $r+s+u-\left(i_{1}-1\right) t>r+s+t$ we have, $r+s+u-i_{1} t>r+s>k r$ and hence as above $G_{I}$ has class $k-I+1$. If further $i_{1}=i_{2}, G_{I}$ splits and so has Wielandt length $k-I+1$, which implies $G$ has Wielandt length $k+1$. If $i_{1}<i_{2}, G_{I}$ has class $k-I+1$ and by Corollary $4.5 G_{I}$ has Wielandt length $k-I+1$, and $G$ has Wielandt length $k+1$.

We now consider the case $r+s \leqslant k r$. Since $G$ has class $k+1$ we have $(k+1) r \geqslant$ $r+s+u>k r$. Let $j_{0}$ be the smallest integer such that $r+s+u-j_{0} t \leqslant k r$. If $j_{0}>I$, then $G_{j}$ has class $k-j+1,0 \leqslant j \leqslant I$. If under these circumstances $I=i_{2}$, then $G_{I}$ splits and the Wielandt length of $G_{I}$ is $k-I+1$, and hence of $G$ is $k+1$. 
If $j_{0}>I$ and $I<i_{2}$, then $G_{I}$ has class $k-I+1$, but by Corollary $4.5 G_{I}$ has Wielandt length $k-I$, and hence $G$ has Wielandt length $k$.

If $j_{0} \leqslant I$, then $G_{j}$ has class $k-j+1,0 \leqslant j<j_{0}$ and class $k-j, j_{0} \leqslant j \leqslant I$. Thus $G_{I}$ has class $k-I$ and either splits, having Wielandt length $k-I$, or by Corollary 4.5 $G_{I}$ has Wielandt length $k-I$. In either case $G$ has Wielandt length $k$, and the proof of the theorem is complete.

\section{METACYCLIC 2-GROUPS}

As mentioned previously the classification of metacyclic 2-groups is more complex than for odd primes. As well as the "ordinary groups" like those already considered for p-odd, we have what King calls the "exceptional metacyclic 2-groups". It has not been easy to find a single form of presentation that is convenient to use, but with some modifications to King [2] and using the work of Newman and Xu (unpublished) it can be deduced that any metacyclic 2-group has a unique presentation in one of the following ways.

$$
G=\left\langle a, b: a^{2^{r+a+u}}=1, b^{2^{r+a+t}}=a^{2^{r+a}}, a^{b}=a^{1+2^{r}}\right\rangle
$$

where

(a) $r \geqslant 2, u, s, t \geqslant 0$ and $u \leqslant r$, or

(b) $r=1, u=0,1, s=0$ and $t \geqslant 1$.

$$
G=\left\langle a, b: a^{2^{r+a}}=1, b^{2^{2+l}}=a^{2^{r+a-1}}, a^{b}=a^{-1+2^{r}}\right\rangle
$$

where

(a) $r \geqslant 2, s \geqslant 1, t \geqslant 1$, or

(b) $r \geqslant 2, s=0, t=1$.

where $r \geqslant 2, s, t \geqslant 0, s+t>0$.

The family of groups presented in (5.1) are the ordinary metacyclic 2-groups, while those in (5.2) and (5.3) are the exceptional metacyclic 2-groups.

\section{The WIELANDT SUBgroup OF A METACYCLIC 2-GROUP}

We need to consider each of the above cases separately. The groups with presentation given by (5.1) are similar to the metacyclic groups for $p$ odd, and all identities and calculations are exactly the same except when $p=2, u=r$ and $t=0$. In this case the identity (2.8)(ii) is no longer true and Theorem 3.1 needs to be stated as follows: 
Theorem 6.1. Let $G$ be a metacyclic 2-group, with presentation as in (5.1), with $u=r$ and $t=0$. Then

$$
\omega(G)=N_{G}(\langle b\rangle) \cap N_{G}\left(\left(b a_{0}^{-1}\right\rangle\right)
$$

where $a_{0}=a^{1+2^{r-1}}$.

Proof: We note that for $w \in N_{G}(\langle b\rangle) \cap N_{G}\left(\left\langle b a_{0}^{-1}\right\rangle\right)$,

(i) $b^{w}=b a^{m 2^{r+d}}$ for some integer $m$,

(ii) $\left(b a_{0}^{-1}\right)^{w}=b a_{0}^{-1}$

(iii) $a_{0}^{w}=a_{0} a^{m 2^{r+s}}$.

As $G=\left\langle b, a_{0}\right\rangle$, any $g \in G$ can be written $g=b^{j}\left(a_{0}\right)^{i}$ and then

$$
g^{w}=g^{1+m 2^{r+\rho}} \text { unless } 2 \mid j \text { and } 2 \nmid i
$$

and $g^{w}=g^{1+m 2^{r+d\left(1+2^{r+1}\right)}}$ if $2 \nmid i$ or $2 \mid j$.

This is sufficient to prove Theorem 6.1.

The main result of Section 3 remains true.

THEOREM 6.2. Let $G$ be a metacyclic 2-group, with presentation as in (5.1). Then

(i) for $0 \leqslant u \leqslant t$

$$
\omega(G)=\left\langle a^{2^{s}}, b^{2^{s+u}}\right\rangle
$$

(ii) for $u>t \geqslant 0$

$$
\omega(G)=\left\langle a^{2^{u-t+s}}, b^{2^{t+s}} a^{-2^{\bullet}}\right\rangle .
$$

The proof is exactly the same as for Theorem 3.2 except when $u=r$ and $t=0$, when a little more care needs to be taken.

We now turn to the exceptional metacyclic 2-groups, that is, groups that can be written with presentation as in (5.2) or (5.3). In all these groups, the following results are true.

(6.3) For any positive integer $i$,

(i) $G^{\prime}=\left\langle a^{2}\right\rangle$

(ii) $[a, b]=a^{-2+2^{r}}$

(iii) $\left[a^{i}, b\right]=[a, b]^{i}$

(iv) $[a, i b]=a^{\left(-2+2^{r}\right)^{i}}$

(v) $(a b)^{2^{i}}=b^{2^{i}} a^{2^{(r+i-1)} \lambda}$ where $2 \nmid \lambda$

(vi) $\zeta(G)=\left\langle a^{2^{r+a-1}}, b^{v}\right\rangle, v=\max \{1, s\}$. 
For the groups with presentations as in (5.2) and (5.3), the calculations are similar to those in Section 3, but rely on the relationships in (6.3). The results of such calculations are summarised below.

(6.4) Let $G$ be a metacyclic 2-group that can be written with presentation as in (5.2). Then

(i) for $t \geqslant r$

$$
\begin{aligned}
\omega(G) & =N_{G}(\langle b\rangle) \cap N_{G}\left(\left\langle b^{2^{2-r+1}} a^{-1}\right\rangle\right) \\
& =\left\langle a^{2^{r+s-2}}, b^{2^{2}}\right\rangle
\end{aligned}
$$

(ii) for $t<r$

$$
\begin{aligned}
\omega(G) & =N_{G}(\langle b\rangle) \cap N_{G}(\langle a b\rangle) \\
& = \begin{cases}\left\langle a^{2^{r+s-2}}, b^{2^{2-1}}\right\rangle & s>1 \\
\left\langle a^{2^{r+c-2}}, b^{2}\right\rangle & s=0,1, r+s>2 \\
G & s=0, t=1, r=2 .\end{cases}
\end{aligned}
$$

In the case (6.4) (ii) above, the Wielandt subgroup is in fact the second centre of the group, and so is as large as it can posssibly be. With $s=0, t=1, r=2$ above, $G$ is the quaternion group and the Wielandt subgroup is the whole of $G$. This is the only case that we consider where the Wielandt subgroup is not abelian.

The groups with presentation given in (5.3) can all be written as splitting extensions. It is easily shown in this case that $\omega(G)=N_{G}(\langle b\rangle) \cap N_{G}(\langle a b\rangle)$ and if $t>0$, then $w \in N_{G}(\langle b\rangle)$ if and only if $w$ centralises $b$, and $w \in N_{G}(\langle a b\rangle)$ if and only if $w$ centralises $a b$. Since $G=\langle b, a b\rangle$, this implies that $\omega(G)=\zeta(G)$. For $t=0$ the calculations are a little more complex, but we have the following results.

(6.5) Let $G$ be a metacyclic 2-group that can be written with presentation as in (5.3). Then
(i) for $t>0, \omega(G)=\zeta(G)= \begin{cases}\left\langle a^{2^{r+s-1}}, b^{2^{t}}\right\rangle & s \neq 0 \\ \left\langle a^{2^{r-1}}, b^{2}\right\rangle & s=0\end{cases}$
(ii) for $t=0, \omega(G)=\left\langle a^{2^{r+a-1}}, b^{2^{a-1}}\right\rangle$.

\section{The Wielandt Length of a Metacyclic 2-group}

If an ordinary metacyclic 2-group has nilpotency class $k+1$, then its Wielandt length is $k$ or $k+1$. This result is proved in exactly the same way as for the corresponding groups when $p$ is an odd prime. Thus we need only consider the exceptional 
metacyclic 2-groups. We consider firstly the groups which can be written as splitting extensions, that is, the groups which can be written with presentation as in (5.3).

We note that these groups have nilpotency class $r+s$, and in all cases the class of $G / \omega(G)$ is $r+s-1$. The factor group is either abelian, or if not can again be written with a presentation as in (5.3). Thus for these groups we can conclude that the class is the same as the Wielandt length.

Looking at those groups that can be written with presentations as in (5.2), we see that such a group is nilpotent of class $r+s$. In this case $G / \omega(G)$ has class $r+s-2$ and is either trivial, or abelian, or if neither of these the factor group can be written with presentation as in (5.3). Thus the Wielandt length of such a group is $r+s-1$, one less than the class of the group.

\section{REFERENCES}

[1] A.R. Camina, 'The Wielandt length of finite groups', J. Algebra 15 (1970), 142-148.

[2] Bruce W. King, 'Presentations of metacyclic groups', Bull. Austral. Math. Soc. 8 (1973), 103-131.

[3] E. Schenkman, 'On the norm of a group', Mlinois J. Math. 4 (1960), 150-152.

[4] M.F. Newman and Xu Mingyao, 'Metacyclic groups of prime-power order', Adv. in Math. 17 (1988), 106-170.

[5] Helmut Wielandt, 'Über den Normalisator der subnormalen Untergruppen', Math. Z. 69 (1958), 463-465.

Department of Mathematics

Faculty of Science

The Australian National University

GPO Box 4

Canberra ACT 2601

Australia 